\title{
Loss of ASAP3 destabilizes cytoskeletal protein ACTG1 to suppress cancer cell migration
}

\author{
YU LUO ${ }^{1,2^{*}}$, FANG KONG ${ }^{2 *}$, ZHEN WANG $^{2 *}$, DAHAN CHEN ${ }^{2}$, QIUYAN LIU $^{3}$, \\ TAO WANG ${ }^{2}$, RUIAN XU ${ }^{3}$, XIANYUAN WANG ${ }^{1}$ and JAMES Y. YANG ${ }^{2,4}$
}

\begin{abstract}
${ }^{1}$ School of Nursing, The Third Military Medical University, Chongqing 400038; ${ }^{2}$ State Key Laboratory of Cellular Stress Biology, Innovation Center for Cell Biology, School of Life Sciences, Xiamen University, Xiang'an, Xiamen, Fujian 361102; ${ }^{3}$ School of Biomedical Sciences, Huaqiao University, Quanzhou, Fujian 362021; ${ }^{4}$ Fujian Provincial Transgenic Core,

Xiamen University Laboratory Animal Center, Xiamen, Fujian 361102, P.R. China
\end{abstract}

Received April 19, 2013; Accepted October 31, 2013

DOI: $10.3892 / \mathrm{mmr} .2013 .1831$

\begin{abstract}
ArfGAP with SH3 domain, ankyrin repeat and PH domain 3 (ASAP3), previously known as ACAP4, DDEFL1 and UPLC1, is considered to be an important regulator in cancer cell migration/invasion and actin-based cytoskeletal remodeling. However, the underlying mechanisms through which ASAP3 mediates these processes are not well-elucidated. This study reported that in certain types of cancer cells, loss of ASAP3 suppressed cell migration/invasion, in part by destabilizing $\gamma$-actin-1 (ACTG1), a cytoskeletal protein considered to be an integral component of the cell migratory machinery, essential for the rearrangement of the dynamic cytoskeletal networks and important in diseases, such as brain malformation, hearing loss and cancer development. The data, for the first time, link ASAP3 with ACTG1 in the regulation of cytoskeletal maintenance and cell motility.
\end{abstract}

\section{Introduction}

The ADP-ribosylation factor GTPase-activating proteins (ArfGAPs) are a family of proteins that catalyze the conversion of the active GTP-bound ADP-ribosylation factors (Arfs) to the inactive GDP-bound Arfs. ArfGAPs are proposed to function in fundamental biological processes, such as secre-

Correspondence to: Professor James Y. Yang, State Key Laboratory of Cellular Stress Biology, Innovation Center for Cell Biology, School of Life Sciences, Xiamen University, Huang Chaoyang Hall, Xiang'an South Road, Xiang'an, Xiamen, Fujian 361102, P.R. China

E-mail: jyqy2008@gmail.com

Professor Xianyuan Wang, School of Nursing, The Third Military Medical University, 30 Gaotanyan Street, Shapingba, Chongqing 400038, P.R. China

E-mail: zhzhang812@yahoo.com.cn

*Contributed equally

Key words: ASAP3, ACTG1, cell migration, cell invasion tion, endocytosis, phagocytosis, cytokinesis, cell adhesion, cell proliferation, cell migration/invasion, membrane trafficking, cytoskeletal remodeling and $\mathrm{Ca}^{2+}$ signaling (1-8). Based on their protein domain structures and phylogeny, ArfGAPs were classified into several subgroups, including ASAPs [ArfGAPs containing Src-homology-3 (SH3), ankyrin (ANK) repeats and plekstrin homology (PH) domain], ACAPs (ArfGAPs containing coiled-coil, ANK repeats and $\mathrm{PH}$ domain) and ARAPs (ArfGAPs containing Rho GAP domain).

Utilizing a genome-wide complementary DNA (cDNA) microarray analysis, Okabe et al (9) identified a novel protein that was highly overexpressed in human hepatocarcinoma tissues. Due to its high amino acid sequence similarity with that of development and differentiation enhancing factor-2, the newly identified protein was termed development and differentiation enhancing factor-like-1 (DDEFL1), which was previously known as upregulated in liver cancer-1 (UPLC1). In addition to liver cancer cells and tissues, significant expression of DDEFL1 was identified in normal lung and liver tissues and leukocytes in humans. Moreover, overexpression of DDEFL1 in two cell lines that have low endogenous expression of DDEFL1 (SNU423 and Alexander human hepatoma cells) appeared to promote cell growth, but antisense RNA-mediated inhibition of DDEFL1 suppressed the growth of hepatoma SNU475 cells (9). Subsequently, Fang et al (10) identified an ArfGAP that utilizes Arf6 as the preferred substrate in HeLa cervical cancer cells, using a proteomic approach. This Arf6-specific GAP was found to contain two coiled coils, one PH domain, one GAP motif and two ANK repeats, therefore, it was termed ACAP4. Notably, the depletion of ACAP4 by small interfering RNA (siRNA), or inhibition of Arf6 GTP hydrolysis by overexpressing GAP-deficient ACAP4, suppressed Arf6-dependent cell migration in wound-healing assays, demonstrating that ACAP4 is required for Arf6-mediated migratory activity in HeLa cells. It was also demonstrated that ACAP4 effectively interacts with ezrin, an important membrane-cytoskeletal linker protein. The formation of ACAP4-ezrin was demonstrated to be important in acid secretion in gastric parietal cells by orchestrating H,K-ATPase-containing tubulovesicular trafficking to the apical plasma membrane (11). It was also shown that 
ACAP4 regulates integrin- $\beta 1$ recycling in epidermal growth factor-stimulated cell migration by interacting with growth factor receptor-binding protein-2 (Grb2) via Tyr-733 phosphorylation (12). Ha et al (13) analyzed the primary sequence and phylogeny of the highly conserved Arf GAP domain of the reported mammalian ArfGAPs and suggested that UPLC1/DDEFL1/ACAP4 is an ASAP-type protein and should be named ASAP3; thus, in the present study, we have decided to use this name. Enzyme characterizations have indicated that ASAP3 is stimulated by phosphatidylinositol 4,5-bisphosphate and is capable of using Arf1, Arf5 and Arf6 as substrates in vitro. ASAP3 has been demonstrated to be associated with focal adhesions and circular dorsal ruffles in MDA-MB-231 breast cancer and U118 glioma cells. ASAP3, however, did not localize with invadopodia in MDA-MB-231 breast cancer cells or podosomes in NIH-3T3 mouse fibroblasts. In MDA-MB-231 cells transfected with plasmids overexpressing either the active or inactive ASAP3, the distribution of vinculin or paxillin in focal adhesions and invadopodia was not affected. However, in cells with reduced ASAP3 protein, a significant reduction in actin-containing stress fibers was observed, although the expression of $\beta$-actin was not significantly affected. With the disruption of the stress fiber network, the level of phosphomyosin was significantly decreased (13). Phosphomyosin is able to bind to the fibers in the network to stabilize them (14). Similarly, reduced ASAP3 levels have been demonstrated to result in slowed migration and invasion in mammary carcinoma cells (13).

Overall, the aforementioned studies suggest that ASAP3 is essential in cytoskeletal remodeling and cancer cell migration/invasion. However, the underlying mechanisms remain to be further investigated. For instance, it is not clear how the stress fibers are disrupted and what particular cytoskeletal actins are affected by ASAP3. The present study reported that ASAP3 mediates cancer cell migration and invasion at least in part by controlling the expression of cytoskeletal $\gamma$-actin-1 (ACTG1) protein.

\section{Materials and methods}

Cell cultures, treatments and transient transfections. H1299 human non-small cell lung cancer cells and HepG2 hepatocellular carcinoma cells were purchased from the American Type Culture Collection (Manassas, VA, USA). The cells were normally maintained in Dulbecco's modified Eagle's medium supplemented with $10 \%$ (v/v) fetal bovine serum, unless indicated otherwise. For glucose or serum depletion treatments, four types (serum free $+5 \mathrm{mM}$ glucose, serum-free $+25 \mathrm{mM}$ glucose, $10 \%$ serum $+5 \mathrm{mM}$ glucose and $10 \%$ serum $+25 \mathrm{mM}$ glucose) of reconstituted DMEM media were prepared and used. Plasmid transfections were performed with Lipofectamine ${ }^{\circledR} 2000$ reagent (Invitrogen Life Technologies, Carlsbad, CA, USA) according to the manufacturer's instructions.

Construction of recombinant adenoviral vectors carrying shRNAs against ASAP3, and of the plasmid overexpressing human ACTG1. Three gene cassettes containing short hairpin RNAs (shRNAs) against ASAP3, as well as a gene cassette containing a scrambled control sequence, were designed using an siRNA Selection Program (http://jura.wi.mit. edu/siRNAext/home.php) and chemically synthesized (Sangong Biotech, Co., Ltd, Shanghai, China). The BamHI- (Cat. \#FD0054)and HindIII [Cat. \#FD0504; Fermentas, Thermo Fisher Scientific (China) Co., Ltd., Beijing, China]-digested (gene cassettes were inserted into the corresponding restriction sites on pSilencer 3.0-H1-neo (Ambion, Austin, TX, USA), generating pSilencer-shRNA-1, -2 and -3 and its control pSilencer-shRNA-CK. Subsequently, DNA fragments containing the shRNA cassettes, as well as the $\mathrm{H} 1$ promoter, were polymerase chain reaction (PCR)-amplified using the four plasmids and the following primers: 5'-ACGGTACCTGATGACGGTGAAAACCTCT-3' (forward) and 5'-GACCTCGAGGGCTTTACACTT TATGCTTCC-3' (reverse). The four KpnI/XhoI-digested PCR-amplified fragments were used for subcloning into the Ad-Track cytomegalovirus (CMV) vector and recombining into the Ad-Easy adenoviral vector using the AdEasy-XL Adenoviral Vector system (Stratagene, Cedar Creek, TX, USA) as instructed by the manufacturer (15), which generated recombinant adenoviruses containing shRNAs against ASAP3 (Ad-shRNA-1, -2 and -3 respectively) and their control (Ad-shRNA-CK).

A 1,128-bp human ACTG1 (GenBank accession no. NM-00119995) cDNA was PCR-amplified from cDNAs prepared from HepG2 human hepatoma cells using the following primers: 5'-CGCGAATTCAGAAGAAGAGATCG CCGC-3' (forward) and 5'-CCCGGATC-CTTAGAAGC ATTTGCGGTG-3' (reverse). The PCR-amplified DNA fragment was digested with EcoRI and HindIII, and subcloned into the pFlag-CMV2 vector (Addgene, Cambridge, MA, USA) to generate a human ACTG1-overexpressing plasmid, pFlag-ACTG1.

Analysis of mRNA expression. Total RNA was extracted from HepG2 or H1299 cells with TRIzol reagent (Invitrogen Life Technologies) according to the manufacturer's instructions. For the analysis of ASAP3 mRNA, semi-quantitative analyses of mRNA expression by reverse transcription (RT)-PCR was conducted according to previously described methods (16), using the following primer pair: 5'-AGCTGAGACATTTGTTCTCTTG-3' (forward) and 5'-TATAAACCAGCTGAGTCCAGAG-3' (reverse). GAPDH served as a control [5'-ACAACAGCCTCAAGATCATCAG-3' (forward) and 5'-GGTCCACCACTGACACGTTG-3' (reverse)] (9).

Western blot analysis of proteins. Lysates from cultured cells were mixed with the radioimmunoprecipitation assay buffer [25 mM Tris- $\mathrm{HCl}, \mathrm{pH} 7.6,150 \mathrm{mM} \mathrm{NaCl}, 1 \% \mathrm{NP}-40$, $1 \%$ sodium deoxycholate, $0.1 \%$ sodium dodecyl sulfate, $1 \mathrm{ml}$ protease inhibitor cocktails (Cat. \#KGP603; Keygene, Nanjing, China) and $5 \mathrm{ml}$ phosphatase inhibitor cocktail (Cat. \#KGP602; Keygene) were added to 1 liter RIPA buffer immediately before use], boiled for $5 \mathrm{~min}$ and sodium dodecyl sulfate-polyacrylamide gel electrophoresis was applied. The protein in the gel was electronically transferred to a nitrocellulose membrane. Following blocking by $5 \%$ skimmed milk solution diluted in Tris-buffered saline Tween 20 (TBST) for $1 \mathrm{~h}$ at room temperature, the membrane was incubated 
A

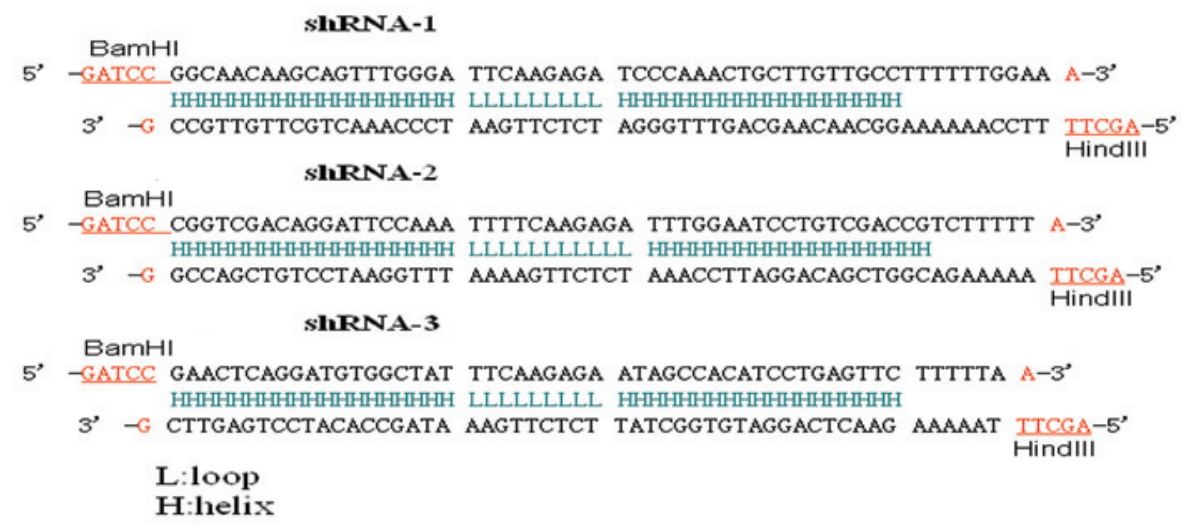

B
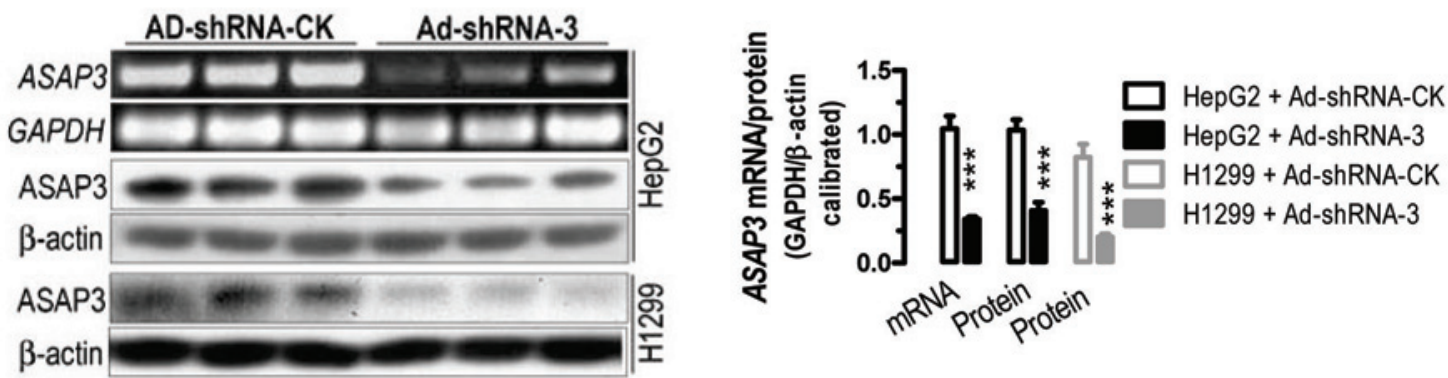

Figure 1. Treatment of human non-small cell lung cancer H1299 cells and hepatocellular carcinoma HepG2 cells with adenoviruses containing ASAP3-shRNAs caused significant downregulation of ASAP3 mRNA and protein. (A) Schematic graphs illustrating the design of three gene cassettes for shRNA against ASAP3. H, nucleotides in the helix regions; L, nucleotides in the loop regions. (B) Gel electrophoresis of RT-PCR-amplified ASAP3 complementary DNA and western blot analysis of ASAP3 protein expression illustrating significant adenovirus-mediated knockdown of ASAP3 in H1299 and HepG2 cells. Cells were infected with the control AD-shRNA-CK or the recombinant Ad-shRNA-3, at a dosage of $\sim 400$ virus particles/cell. The cell cultures were incubated at $37^{\circ} \mathrm{C}$ for $12 \mathrm{~h}$ and then the medium was replaced with virus-free medium. Following $48 \mathrm{~h}$ of further incubation, cells were collected for ASAP3 mRNA and protein analyses. ${ }^{* * * *} \mathrm{P}<0.001, \mathrm{n}=3$. shRNA, short hairpin RNA; ASAP3, ArfGAPs containing Src-homology-3, ankyrin repeats and plekstrin homology domain 3; GAPDH, glyceraldehyde-3-phosphate dehydrogenase.

with the primary antibody (diluted 1:1,000 with coating buffer) solutions overnight at $4^{\circ} \mathrm{C}$ and washed with TBST three times. The washed membrane was further incubated with secondary antibody in TBST for $1 \mathrm{~h}$ at room temperature. The primary antibodies used were rabbit polyclonal anti-Flag antibody (Octa-probe antibody, Cat. \#sc-807, 1:1,000 dilution; Santa Cruz Biotechnology, Inc., Dallas, TX, USA), rabbit polyclonal anti-ASAP3/DDEFL1/UPLC1 antibody (Cat. \#200-401-990, 1:1,000 dilution; Rockland Immunochemicals Inc., Gilbertsville, PA, USA), mouse monoclonal anti-ACTG1 (1-24) antibody (Cat. \#sc-65635, 1:1,000 dilution; Santa Cruz Biotechnology, Inc.), mouse monclonal anti- $\beta$-actin (C4) antibody (Cat. \#sc47778, 1:1,000 dilution; Santa Cruz Biotechnology, Inc.) and mouse monoclonal anti- $\alpha$-tubulin antiody (Cat. \#5286, 1:1,000 dilution; Santa Cruz Biotechnology, Inc.). Detection was achieved using the Immobilon Western Chemiluminescent Horseradish Peroxidase Substrate kit (Millipore, Billerica, MA, USA). The western blot images were captured by the Biosense SC8108 Gel Documentation system with GeneScope V1.73 software (Shanghai BioTech, Shanghai, China). The digital density values were acquired by Image-Pro Plus 6.0 software (MediaCybernetics, Rockville, MD, USA).

Cell proliferation, wound-healing and transwell assays. For the cell proliferation assays, a total density of $4 \times 10^{3}$ cells were seeded in 96-well plates and infected with the recombinant adenoviruses at a dosage of $\sim 400$ viral particles/cell. After 48 h, $20 \mu \mathrm{l}$ of 3-(4,5-dimethylthiazol-2-yl)-2,5-diphenyl-tetrazolium bromide (MTT; $5 \mathrm{mg} / \mathrm{ml}$ ) was applied to each well and incubated for a further $4 \mathrm{~h}$. Cells were lysed with the addition of acidic isopropyl alcohol (containing $0.04 \mathrm{M} \mathrm{HCl}$ ) or dimethylsulfoxide for $15 \mathrm{~min}$. The absorbance was measured at $570 \mathrm{~nm}$ using a POLARstar Omega microplate reader (BMG Labtech, Ortenberg, Germany).

For the cell migration assays, recombinant adenovirus-infected cells or plasmid-transfected cells were marred with a linear scratch by a sterile pipette tip. Wound healing was recorded by photographing at specified time points following the scratch. In addition, cell migration was analyzed by the transwell assays (BD Biosciences, Sparks, MD, USA) according to the manufacturer's instructions. The comparison of the rates of cell migration was achieved by direct visualization of the recorded images.

Proteomic analyses of differentially expressed proteins by two-dimensional gel electrophoresis and mass spectrometry. H1299 cells were cultured and infected by recombinant adenovirus containing either pAd-shRNA-3 or pAd-shRNA-CK for $72 \mathrm{~h}$. The cell lysates were collected for proteomic analyses by two-dimensional gel electrophoresis and mass spectrometry analyses (4800 Plus MALDI-TOF/ 

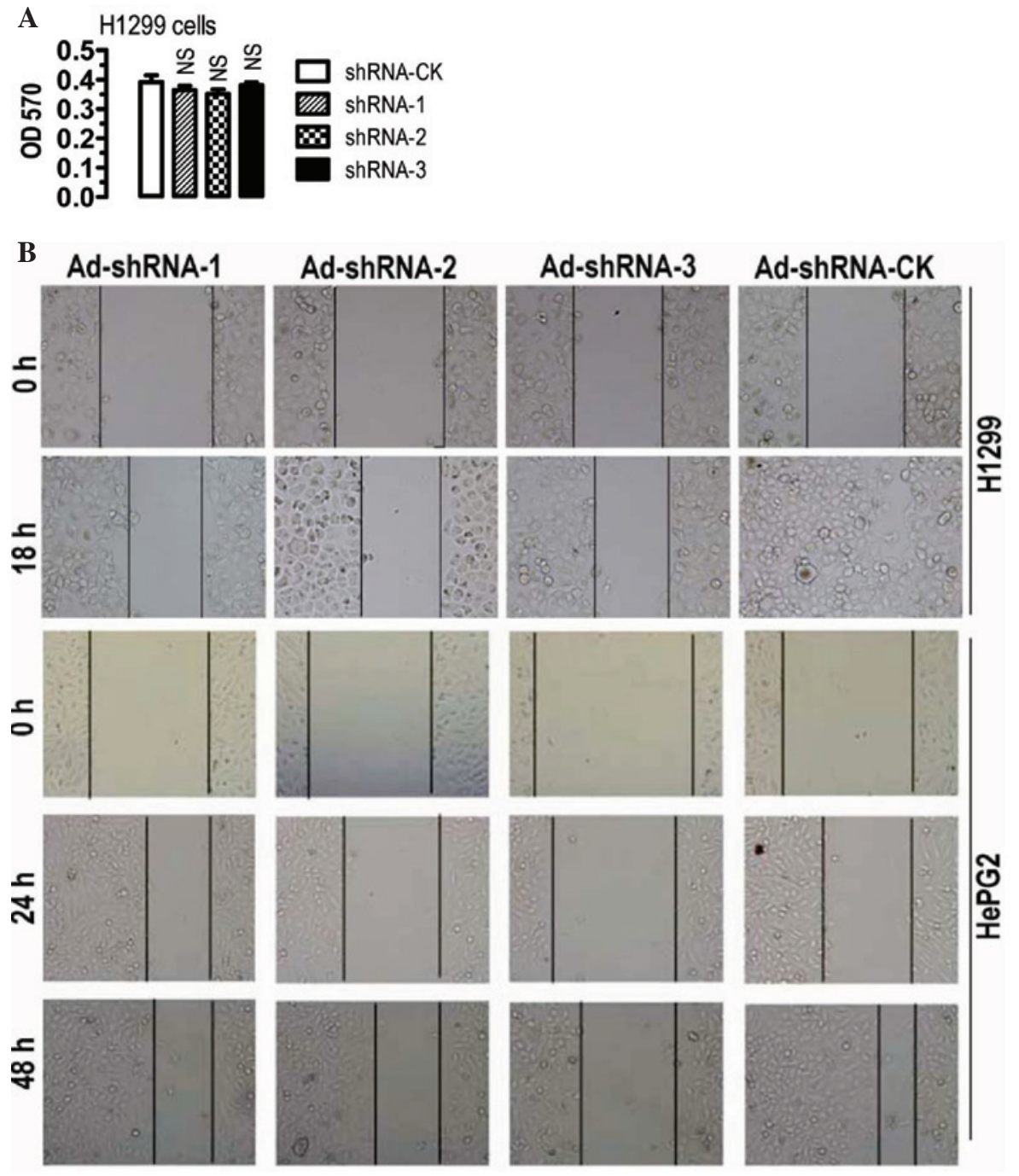

Figure 2. Adenovirus-mediated ASAP3 knockdown suppressed cell migration in H1299 human non-small cell lung cancer cells and HepG2 hepatocellular carcinoma cells, but did not affect cell proliferation in H1299 cells. (A) Effects of adenovirus-mediated ASAP3 knockdown on cellular proliferation of H1299 cells. An appropriate number of cells were plated in 96-well plates. MTT $(20 \mu 1,5 \mathrm{mg} / \mathrm{ml})$ was applied to each well $48 \mathrm{~h}$ after viral infection and incubated for a further $4 \mathrm{~h}$. The cells were lysed with the addition of acidic isopropyl alcohol (containing $0.04 \mathrm{M} \mathrm{HCl}$ ) overnight. The absorbance was measured at $570 \mathrm{~nm}$ with a microplate reader. NS, not significant; $\mathrm{n}=3$. (B) Effects of adenovirus-mediated ASAP3 knockdown on cell migration as determined by wound-healing assays. The H1299 and HepG2 cells were infected with adenoviruses containing short hairpin RNAs against ASAP3 or their control, and at the dosage of $\sim 400$ viral particles/cell. Wound healing was recorded by photographing at specified time points. Results were representative of three independent experiments. OD, optical density; shRNA, short hairpin RNA; ASAP3, ArfGAPs containing Src-homology-3, ankyrin repeats and plekstrin homology domain 3.

TOF ${ }^{\mathrm{TM}}$ Analyzer; Applied Biosystems, Foster City, CA, USA), that were performed by the Beijing Proteome Research Center (Beijing, China).

Statistical analyses. Numerical data were analyzed by Graphpad Prism 5.0 (GraphPad Software, La Jolla, CA, USA) and expressed as the means \pm standard error of the mean. One-way analysis of variance with Bonferroni's post hoc test was used for multiple comparisons and Student's t-test (two-tailed) was used for pair-wise comparisons.

\section{Results}

Adenovirus-mediated ASAP3 knockdown inhibits cancer cell migration but does not affect cell proliferation or the cell cycle. Based on bioinformatics analyses, four gene cassettes were designed, with three cassettes carrying shRNAs against human ASAP3 (GenBank accession no. NM-017707) (Fig. 1A)

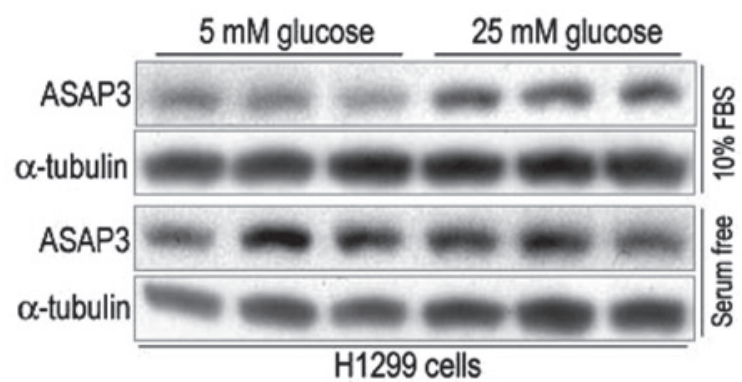

Figure 3. Effects of serum and glucose concentration on ASAP3 protein expression in $\mathrm{H} 1299$ cells, as determined by western blot analysis. ASAP3, ArfGAPs containing Src-homology-3, ankyrin repeats and plekstrin homology domain 3; FBS, fetal bovine serum.

and one cassette carrying a scrambled control sequence. The four chemically synthesized gene cassettes were used to create four recombinant adenoviruses carrying the 
A

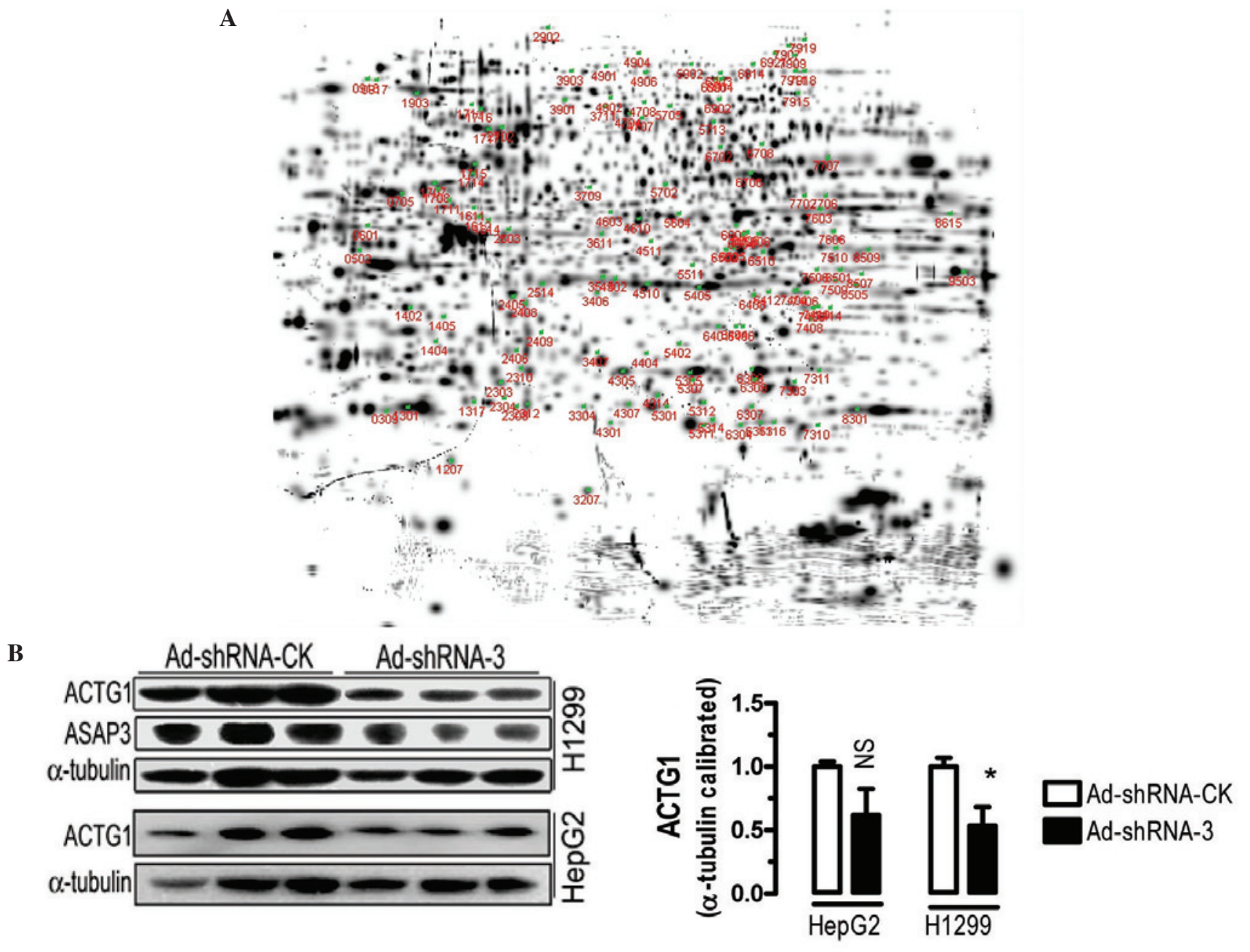

Figure 4. ASAP3 knockdown is associated with significant downregulation in the expression of cytoskeletal protein ACTG1. (A) Differentially expressed proteins between Ad-shRNA-3 infected H1299 cells and Ad-shRNA-CK infected cells as analysed by the two-dimensional gel electrophoresis. (B) Effects of adenovirus-mediated ASAP3 knockdown on ACTG1 protein expression in $\mathrm{H} 1299$ and HepG2 hepatocellular carcinoma cells. ${ }^{*} \mathrm{P}<0.05$, n=3. NS, not significant; shRNA, short hairpin RNA; ACTG1, $\gamma$-actin-1; ASAP3, ArfGAPs containing Src-homology-3, ankyrin repeats and plekstrin homology domain 3.

corresponding shRNA (Ad-shRNA-1, -2 and -3) and their control (Ad-shRNA-CK). The infection studies demonstrated that infection with Ad-shRNA-1, -2 (data not shown) and -3 significantly reduced the mRNA and protein levels of ASAP3 in cultured H1299 and HepG2 cancer cells, compared with those in cells infected with Ad-shRANA-CK (Fig. 1B). For instance, in comparison with cells infected with Ad-shRNA-CK, infection with Ad-shRNA-3 caused a significant reduction of $\sim 64 \%$ in ASAP3 mRNA expression in the HepG2 cells $(\mathrm{P}<0.001)$, as determined by semi-quantitative RT-PCR analyses. Simililarly, infection with Ad-shRNA-3 led to 60 and $80 \%$ reductions in ASAP3 protein expression in HepG2 and H1299 cells, respectively $(\mathrm{P}<0.001)$. The results demonstrated the successful creation of recombinant viruses carrying shRNAs against ASAP3.

To determine the possible effects of adenovirus-mediated ASAP3 knockdown on cancer cells, cell proliferation, the cell cycle and cell migration in H1299 and HepG2 cells were analyzed. ASAP3 knockdown in H1299 cells did not significantly affect the rate of cell proliferation (Fig. 2A) and neither did the cell cycle appear to be affected (data not shown). By contrast, ASAP3 knockdown appeared to signifi- cantly reduce the likelihood of cell migration in H1299 and HepG2 cells (Fig. 2B). The results, therefore, are consistent with the notion that ASAP3 is an important regulator of cell migration.

Effects of glucose and serum concentration on protein expression of ASAP3. Okabe et al (9) demonstrated that ASAP3 may have growth-promoting ability, particularly when under stressed conditions. Therefore the present study aimed to determine whether low glucose or serum deprivation affect ASAP3 expression. As shown in Fig. 3, among the four treatment groups, H1299 cells grown in media containing 10\% serum and $5 \mathrm{mM}$ glucose had the lowest level of ASAP3 protein expression. In comparison with this, ASAP3 expression appeared to be significantly increased in cells grown in media containing $10 \%$ serum and $25 \mathrm{mM}$ glucose, while H1299 cells grown in media that were serum free also had high levels of ASAP3 protein expression, compared with that of H1299 cells grown in media containing $10 \%$ serum and $5 \mathrm{mM}$ glucose. These results suggest that high glucose and serum deprivation are both capable of inducing ASAP3 protein expression in H1299 cells. 

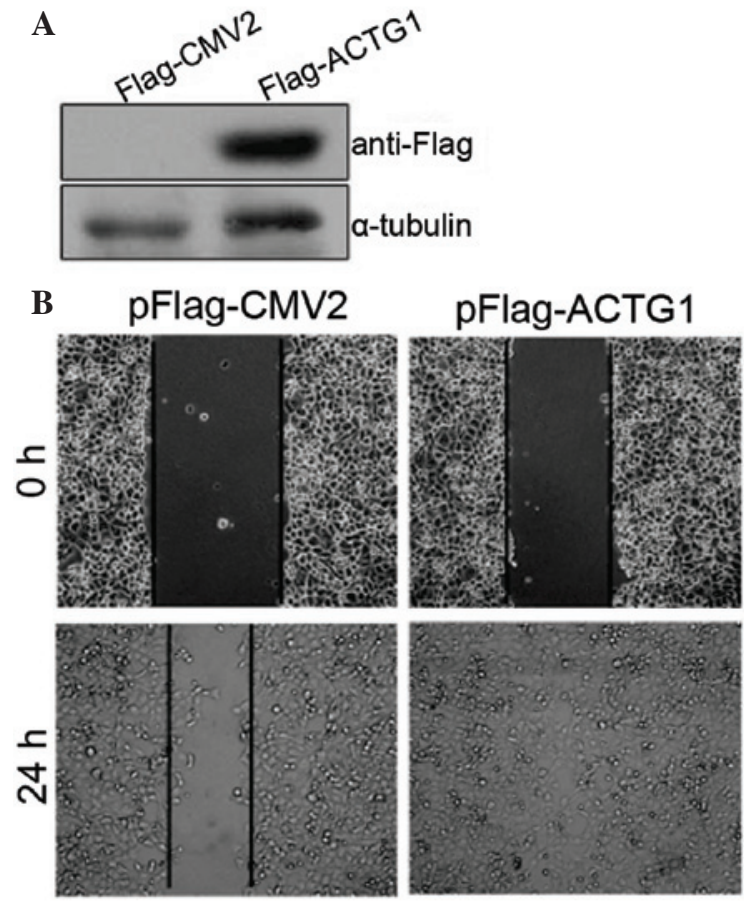

C pFlag-CMV2

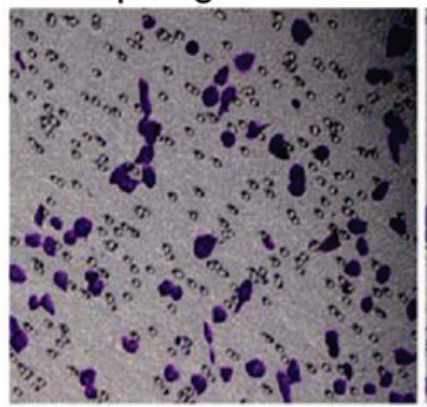

pFlag-ACTG1

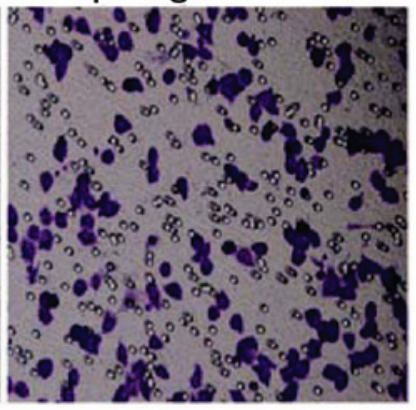

Figure 5. Overexpression of human ACTG1 enhanced wound-healing migration and transwell migration of human non-small cell lung cancer H1299 cells. (A) Upregulation of human ACTG1 in H1299 cells as assayed by western blot analysis. H1299 cells seeded on 6-well plates were transfected with pFlag-CMV2 or Flag-ACTG1 and harvested $24 \mathrm{~h}$ later for protein analyses. (B) Overexpression of human ACTG1 in H1299 cells promotes cell migration as assayed by the wound-healing assays. The H1299 cells seeded on 6-well plates were transfected with pFlag-CMV2 and pFlag-ACTG1 to confluency. Cells were scratched with a sterile pipette tip and phase contrast images of cells were captured either immediately after wounding or $24 \mathrm{~h}$ after wounding. (C) Overexpression of human ACTG1 in H1299 cells promotes cell migration as assayed by the transwell assays. H1299 cells seeded on 6-well plates were transfected with pFlag-CMV2 and pFlag-ACTG1. Following $12 \mathrm{~h}$, cells were harvested and $1 \times 10^{5}$ cells were applied to transwell upper chambers. The lower compartments of the transwells were filled with Dulbecco's modified Eagle's medium containing $10 \%$ fetal bovine serum bait. Following $24 \mathrm{~h}$ of incubation, cells that migrated out onto the lower surface of the membrane were stained in $0.1 \%$ crystal violet and photographed. CMV, cytomegalovirus; ACTG1, $\gamma$-actin-1.

ASAP3 may regulate cell migration through controlling the stability of cytoskeletal protein ACTG1. The demonstration that ASAP3 controlled cell migration in H1299 and HepG2 cells is largely consistent with previous findings in other cancer cell lines, including the HeLa cell line $(10,13)$. However, the underlying mechanisms were not clear. Utilizing two-dimensional gel electrophoresis and mass spectrometry analyses, proteomic analyses were performed with H1299 cells infected with Ad-shRNA-3 and Ad-shRNA-CK. The two-dimensional gel analyses identified 124 protein spots that were differentially expressed by at least two-fold between the Ad-shRNA-3- and Ad-shRNA-CK-transfected cells. Protein spot no. 1207 was significantly downregulated (Fig. 4A) and spot no. 8507 was significantly upregulated compared with the control (with the expression of Ad-shRNA-CK transfected cells being 1, the expression of Ad-shRNA-3 transfected cells for no. 1207 corresponding spot was 0.11 and no. 8507 corresponding spot was 10.34). Mass spectrometry analyses indicated that the two proteins were non-muscle cytoskeletal proteins, ACTG1 and GAPDH, respectively.

To verify the regulation of ACTG1 by ASAP3, protein expression analyses were performed in H2199 and HepG2 cells. Adenovirus-mediated knockdown of ASAP3 caused downregulation of ACTG1 protein expression in the two cell lines compared with the control, although the difference was only statistically significant in the H1299 cells (Fig. 4B). These results suggested that ASAP3 may be able to regulate the stability or expression of ACTG1.

Overexpression of ACTG1 enhances cancer cell migration. To determine the potential effects of ACTG1 on cancer cells, wound-healing and transwell assays were performed. Notably, overexpression of ACTG1 (Fig. 5A) significantly enhanced the likelihood of cell migration as assayed by wound-healing (Fig. 5B) and transwell (Fig. 5C) assays in the H1299 cells. Similar results were observed for HepG2 cells (data not shown). Together these results suggested that ASAP3 may regulate cell migration at least in part by downregulating the expression ACTG1, thereby disrupting the cytoskeletal fiber networks.

\section{Discussion}

Previous studies have suggested that ASAP3 is pivotal in cell migration $(10,12,13)$. However, the mechanisms through which ASAP3 mediates cell migration were not clearly elucidated. Although, Yu et al (12) demonstrated that ASAP3 may cooperate with Grb2 to regulate integrin $\beta 1$ recycling in cancer cell migration. Grb2 is an adaptor protein important for intracellular signal transduction, cell proliferation, actin-based cell motility and endocytic trafficking, whereas integrins are transmembrane receptors that are important for cell signaling and the regulation of the cell cycle, shape and motility. Despite this, there may be other mechanisms involved. In breast cancer MDA-MB-231 cells, Ha et al (13) demonstrated that overexpression of wild-type ASAP3 or of a GAP-inactive mutant ASAP3, or ASAP3 knockdown did not affect the distribution of vinculin or paxillin, suggesting that ASAP3 does not affect focal adhesions. ASAP3 knockdown, however, was demonstrated to significantly reduce actin stress fibers, although it is not clear which particular actins may be affected. Notably, using proteomic and other approaches in the present study, cytoskeletal ACTG1 was identified as one of the actins directly targeted by ASAP3 in H1299 lung cancer cells and appeared to contribute significantly to $A S A P 3$ knockdown-induced disruption of the actin stress fibers. 
Actins are highly conserved proteins that are involved in cell motility and maintenance of the cytoskeleton, among other functions (17). In vertebrates, three main groups of actin isoforms, namely $\alpha, \beta$ and $\gamma$, have been identified. The $\alpha$-actins are found in muscle tissues and are a major constituent of the contractile apparatus. Conversely the $\beta$ - and $\gamma$-actins, co-exist in the majority of cell types as components of the cytoskeleton, and as mediators of internal cell motility. A previous study associated alterations in the expression of ACTG1 with diseases, such as hearing loss and cancer (18). In auditory cells, multiple mutations in ACTGl were demonstrated to be associated with dominant progressive deafness (19-26). Certain studies have demonstrated that ACTG1 is important for cytoskeletal maintenance but not for development $(27,28)$. However, a more recent study suggested that a de novo mutation in ACTG1 causes a form of brain malformation in humans (Baraitser-Winter syndrome) (29). Moreover, a study in ACTG1 null mice suggested that ACTG1 deficiency led to growth impairment and reduced cell viability (30). Using oligonucleotide microarrays, Sun et al (31) demonstrated that a group of microtubule proteins and intermediate filament proteins, including ACTG1, were significantly upregulated in the liver tissues of transgenic mice overexpressing the oncogenic hepatitis B virus $\mathrm{X}$ protein $(\mathrm{HBx})$, suggesting that the dysregulation of ACTG1 and/or other cytoskeletal proteins may contribute significantly to $\mathrm{HBx}$-induced hepatocarcinogenesis.

Notably, Shum et al (32) demonstrated that in SH-EP neuroblastoma cells, ACTG1 knockdown resulted in a significant decrease in wound healing and transwell migration. In contrast to ASAP3 knockdown in MDA-MD-231 cells, however, there was a significant increase in the size and number of paxillin-containing focal adhesions, coupled with a significant decrease in phosphorylated paxillin in ACTG1 knockdown neuroblastoma cells. It is not clear whether this effect of ACTG1 knockdown is cell type-specific. In addition, inhibition of the Rho-associated kinase (ROCK) with the inhibitor Y-27632 restored the ability of ACTG1-knockdown cells to migrate, suggesting that ACTG1 is an upstream regulator of ROCK-mediated cell migration. More recently, the same research group further demonstrated that ACTG1 can modulate microtubule dynamics (33). Largely consistent with the two aforementioned studies, the present study demonstrated that overexpression of ACTG1 enhanced cancer cell migration in the H1299 and HepG2 cells (Fig. 5). Concordant with a previous suggestion that ArfGAPs may function as upstream regulators of Rho family proteins (1), the results of the present study indicate that in cancer cells, ASAP3 may positively regulate ACTG1 to promote cytoskeletal remodeling and cell migration, particularly ROCK signaling pathway-mediated cell migration (34). At present, however, whether ASAP3 regulates ACTG1 directly or indirectly and the underlying mechanisms are not clear and awaits further investigation. Our demonstration that ASAP3 controls cell migration at least in part by destabilizing cytoskeletal protein ACTG1, may aid future development of drugs aiming at cancer cell migration, invasion and metastasis.

\section{Acknowledgements}

This study was supported in part by grants from the 973 Program, China (grant no. 2009CB941601), the National
Natural Science Foundation of China (grant no. 31271239), the Fujian Provincial Department of Science and Technology (grant no. 2010L0002) and the Open Research Fund of State Key Laboratory of Cellular Stress Biology, Xiamen University (grant no. SKLCSB2012KF005).

\section{References}

1. Randazzo PA, Inoue H and Bharti S: Arf GAPs as regulators of the actin cytoskeleton. Biol Cell 99: 583-600, 2007.

2. Spang A, Shiba Y and Randazzo PA: Arf GAPs: gatekeepers of vesicle generation. FEBS Lett 584: 2646-2651, 2010.

3. Randazzo PA and Hirsch DS: Arf GAPs: multifunctional proteins that regulate membrane traffic and actin remodelling. Cell Signal 16: 401-413, 2004.

4. D'Souza-Schorey C and Chavrier P: ARF proteins: roles in membrane traffic and beyond. Nat Rev Mol Cell Biol 7: 347-358, 2006.

5. Bharti S, Inoue H, Bharti K, Hirsch DS, Nie Z, Yoon HY, Artym V, Yamada KM, Mueller SC, Barr VA and Randazzo PA: Src-dependent phosphorylation of ASAP1 regulates podosomes. Mol Cell Biol 27: 8271-8283, 2007.

6. Randazzo PA, Andrade J, Miura K, Brown MT, Long YQ, Stauffer S, Roller P and Cooper JA: The Arf GTPase-activating protein ASAP1 regulates the actin cytoskeleton. Proc Natl Acad Sci USA 97: 4011-4016, 2000.

7. de Curtis I: Cell migration: GAPs between membrane traffic and the cytoskeleton. EMBO Rep 2: 277-281, 2001.

8. Ismail SA, Vetter IR, Sot B and Wittinghofer A: The structure of an Arf-ArfGAP complex reveals a $\mathrm{Ca}^{2+}$ regulatory mechanism. Cell 141: 812-821, 2010.

9. Okabe H, Furukawa Y, Kato T, Hasegawa S, Yamaoka Y and Nakamura Y: Isolation of development and differentiation enhancing factor-like 1 (DDEFL1) as a drug target for hepatocellular carcinomas. Int J Oncol 24: 43-48, 2004.

10. Fang Z, Miao Y, Ding X, Deng H, Liu S, Wang F, Zhou R, Watson $\mathrm{C}, \mathrm{Fu} \mathrm{C}, \mathrm{Hu} \mathrm{Q}$, et al: Proteomic identification and functional characterization of a novel ARF6 GTPase-activating protein, ACAP4. Mol Cell Proteomics 5: 1437-1449, 2006.

11. Ding X, Deng H, Wang D, Zhou J, Huang Y, Zhao X, Yu X, Wang M, Wang F, Ward T, et al: Phospho-regulated ACAP4-Ezrin interaction is essential for histamine-stimulated parietal cell secretion. J Biol Chem 285: 18769-18780, 2010.

12. Yu X, Wang F, Liu H, Adams G, Aikhionbare F, Liu D, Cao X, Fan L, Hu G, Chen Y, et al: ACAP4 protein cooperates with Grb2 protein to orchestrate epidermal growth factor-stimulated integrin $\beta 1$ recycling in cell migration. J Biol Chem 286: 43735-43747, 2011

13. Ha VL, Bharti S, Inoue H, Vass WC, Campa F, Nie Z, de Gramont A, Ward Y and Randazzo PA: ASAP3 is a focal adhesion-associated Arf GAP that functions in cell migration and invasion. J Biol Chem 283: 14915-14926, 2008.

14. Pellegrin S and Mellor H: Actin stress fibres. J Cell Sci 120: 3491-3499, 2007.

15. He TC, Zhou S, da Costa LT, Yu J, Kinzler KW and Vogelstein B: A simplified system for generating recombinant adenoviruses. Proc Natl Acad Sci USA 95: 2509-2514, 1998.

16. Qiu L, Wu X, Chau JF, Szeto IY, Tam WY, Guo Z, Chung SK, Oates PJ, Chung SS and Yang JY: Aldose reductase regulates hepatic peroxisome proliferator-activated receptor $\alpha$ phosphorylation and activity to impact lipid homeostasis. J Biol Chem 283: 17175-17183, 2008.

17. Herman IM: Actin isoforms. Curr Opin Cell Biol 5: 48-55, 1993.

18. Chou CC, Davis RC, Fuller ML, Slovin JP, Wong A, Wright J, Kania S, Shaked R, Gatti RA and Salser WA: Gamma-actin: unusual mRNA 3'-untranslated sequence conservation and amino acid substitutions that may be cancer related. Proc Natl Acad Sci USA 84: 2575-2579, 1987.

19. Zhu M, Yang T, Wei S, DeWan AT, Morell RJ, Elfenbein JL, Fisher RA, Leal SM, Smith RJ and Friderici KH: Mutations in the $\gamma$-actin gene (ACTG1) are associated with dominant progressive deafness (DFNA20/26). Am J Hum Genet 73: 1082-1091, 2003

20. van Wijk E, Krieger E, Kemperman MH, De Leenheer EM, Huygen PL, Cremers CW, Cremers FP and Kremer H: A mutation in the gamma actin 1 (ACTG1) gene causes autosomal dominant hearing loss (DFNA20/26). J Med Genet 40: 879-884, 2003. 
21. de Heer AM, Huygen PL, Collin RW, Oostrik J, Kremer H and Cremers CW: Audiometric and vestibular features in a second Dutch DFNA20/26 family with a novel mutation in ACTG1. Ann Otol Rhinol Laryngol 118: 382-390, 2009.

22. Liu P, Li H, Ren X, Mao H, Zhu Q, Zhu Z, Yang R, Yuan W, Liu J, Wang $\mathrm{Q}$ and Liu M: Novel ACTG1 mutation causing autosomal dominant non-syndromic hearing impairment in a Chinese family. J Genet Genomics 35: 553-558, 2008

23. Morin M, Bryan KE, Mayo-Merino F, Goodyear R, Mencia A, Modamio-Hoybjor S, del Castillo I, Cabalka JM, Richardson G, Moreno $\mathrm{F}$, et al: In vivo and in vitro effects of two novel gamma-actin (ACTG1) mutations that cause DFNA20/26 hearing impairment. Hum Mol Genet 18: 3075-3089, 2009.

24. Rendtorff ND, Zhu M, Fagerheim T, Antal TL, Jones M, Teslovich TM, Gillanders EM, Barmada M, Teig E, Trent JM, et al: A novel missense mutation in ACTG1 causes dominant deafness in a Norwegian DFNA20/26 family, but ACTG1 mutations are not frequent among families with hereditary hearing impairment. Eur J Hum Genet 14: 1097-1105, 2006.

25. Perrin BJ, Sonnemann KJ and Ervasti JM: $\beta$-actin and $\gamma$-actin are each dispensable for auditory hair cell development but required for Stereocilia maintenance. PLoS Genet 6: e1001158, 2010.

26. Kruth KA and Rubenstein PA: Two deafness-causing (DFNA20/26) actin mutations affect Arp2/3-dependent actin regulation. J Biol Chem 287: 27217-27226, 2012

27. Belyantseva IA, Perrin BJ, Sonnemann KJ, Zhu M, Stepanyan R, McGee J, Frolenkov GI, Walsh EJ, Friderici KH, Friedman TB and Ervasti JM: $\gamma$-actin is required for cytoskeletal maintenance but not development. Proc Natl Acad Sci USA 106: 9703-9708, 2009.
28. Sonnemann KJ, Fitzsimons DP, Patel JR, Liu Y, Schneider MF, Moss RL and Ervasti JM: Cytoplasmic $\gamma$-actin is not required for skeletal muscle development but its absence leads to a progressive myopathy. Dev Cell 11: 387-397, 2006.

29. Riviere JB, van Bon BW, Hoischen A, Kholmanskikh SS, O'Roak BJ, Gilissen C, Gijsen S, Sullivan CT, Christian SL, Abdul-Rahman OA, et al: De novo mutations in the actin genes ACTB and ACTG1 cause Baraitser-Winter syndrome. Nat Genet 44: 440-444, S1-S2, 2012.

30. Bunnell TM and Ervasti JM: Delayed embryonic development and impaired cell growth and survival in Actg1 null mice. Cytoskeleton (Hoboken) 67: 564-572, 2010.

31. Sun Q, Wang Y, Zhang Y, Liu F, Cheng X, Hou N, Zhao X and Yang X: Expression profiling reveals dysregulation of cellular cytoskeletal genes in $\mathrm{HBx}$-induced hepatocarcinogenesis. Cancer Biol Ther 6: 668-674, 2007.

32. Shum MS, Pasquier E, Po'uha ST, O'Neill GM, Chaponnier C, Gunning PW and Kavallaris M: $\gamma$-Actin regulates cell migration and modulates the ROCK signaling pathway. FASEB J 25: 4423-4433, 2011.

33. Po'uha ST, Honore S, Braguer D and Kavallaris M: Partial depletion of gamma-actin suppresses microtubule dynamics. Cytoskeleton (Hoboken) 70: 148-160, 2013.

34. Patel RA, Liu Y, Wang B, Li R and Sebti SM: Identification of novel ROCK inhibitors with anti-migratory and anti-invasive activities. Oncogene: Feb 11, 2013 (Epub ahead of print). 